ROBYN PIERCE and KAYE STACEY

\title{
A FRAMEWORK FOR MONITORING PROGRESS AND PLANNING TEACHING TOWARDS THE EFFECTIVE USE OF COMPUTER ALGEBRA SYSTEMS
}

\begin{abstract}
This article suggests a framework to organise a cluster of variables that are associated with students' effective use of computer algebra systems (CAS) in mathematics learning. Based on a review of the literature and from the authors' own teaching experience, the framework identifies the main characteristics of students' interactions with CAS technology and how these may be used to monitor students' developing use of CAS; from this, the framework may be used to plan teaching in order to gain greater benefit from the availability of CAS. Four case studies describing students' development over a semester are reported. These demonstrate a variety of combinations of technical competencies and personal attributes. They indicate the importance of both the technical and personal aspects but suggest that negative attitudes rather than technical difficulties can limit the effective use of CAS. Finally practical suggestions are given for teaching strategies which may promote effective use of CAS.
\end{abstract}

KEY WORDS: Computer Algebra System, technology use, instrument, technical, attitude, teaching

\section{INTRODUCTION}

Since their development in the 1970s and their introduction into tertiary teaching in the 1980s, the powerful technology of Computer Algebra Systems (CAS) has been recognised as highly valuable for doing mathematics and as potentially valuable for teaching and learning mathematics. Studies involving both tertiary and secondary mathematics classes (for example Heid, 1988; Atkins et al., 1995; Pierce, 1999a; Lagrange, 1999a) have supported the contention that the symbolic manipulation features of CAS can free students from manipulation errors and thus allow them to 
quickly generate both exact and approximate results. They have also demonstrated that the "scaffolding" provided by CAS, as discussed by Kutzler (1994), allows students to handle more complicated problems than most students can do by hand at the same age or stage of mathematics learning. Furthermore, the addition of symbolic manipulation facilities to software that already has both numerical and graphical capabilities can encourage students to make a habit of using these three representations to enhance their understanding (Pierce, 1999b).

The studies listed above also make clear that the mere presence of CAS in a classroom does not mean that its potential benefits will be realised. Students need to learn to operate the technology effectively and to integrate it with their repertoire of techniques for doing and learning mathematics. As Guin and Trouche (1999) carefully document, this is unlikely to be achieved without acknowledgement in the curriculum of the magnitude and importance of the task of integrating CAS with other mathematical practices, nor without the full involvement of teachers. This paper is concerned with how effective teaching practice can be developed. We appreciate that teachers will not judge that using CAS is appropriate in all parts of all courses. We are arguing in the context where using CAS has been judged to be appropriate, in which case students, and teachers, should make the best use of it they can.

In this paper we propose a framework which organises and highlights key cognitive and affective issues in a form which may be helpful for both planning teaching and monitoring the progress of students using CAS technology for mathematics. Our interest is especially in those aspects where interaction between mathematical practices and CAS technology is strongest. These are discussed through examples drawn from the experience of teaching an introductory undergraduate functions and calculus course in which the use of CAS was modelled and encouraged. 
The progress of all members of the first author's 1999 class was closely monitored, and the experiences of four students are here discussed in detail. (The study is fully reported in Pierce, 2002). These students' responses to using CAS were highly individual, with two using CAS to good advantage and two gaining significantly less. Whilst we are aware that experienced teachers can make broad judgements about their students' level of success without any particular framework, we claim that the framework we are proposing provides a structure for the detailed analysis of students' progress, and the responses that teachers should take.

The framework we present was developed following the approach used by McIntosh et al. (1992) to define and explicate the useful but slippery concept of number sense, based on what those authors perceived as the critical variables that need to be studied. The number sense framework was developed in response to the growing prevalence of new technology - in that case the four-function calculator. Our aims for a framework for effective use of CAS are similar. The rationale for our choice of critical variables is informed by the literature and by our own teaching experiences. The components of our framework for CAS could be applied to considering the use of other mathematical software, and this is briefly discussed at the end of the paper.

\section{ASPECTS OF THE EFFECTIVE USE OF CAS}

In general terms, making effective use of a technology such as CAS in a mathematics classroom has both cognitive and affective aspects. The personal (affective) aspect reflects the response of students to the availability of CAS technology. To benefit from the availability of CAS, students must be willing to use it, and be discriminating in its use; the discussion below of the personal aspect in the framework is organised around these two elements. Many factors contribute to an 
individual's personal response to the availability of CAS, including their confidence with computers, their beliefs about what mathematics is most important to learn, the acceptability of CAS in assessment and, as shown by Kendal and Stacey (2001), how the teacher privileges the use of CAS within the course. However, the purpose of this paper is not to describe the causes of (in)appropriate attitudes and beliefs but to provide a simple framework which identifies key components that should be monitored.

The cognitive aspects of effective use of CAS need careful delineation. Using a CAS to do mathematics requires traditional mathematical knowledge, knowledge of the machine and, as many studies have now documented (see below), it also requires a constant interplay of mathematical knowledge and knowledge about the technology. Therefore, the knowledge and skills required for using CAS to solve mathematical problems can be thought of along a continuum - see Figure 1. At one extreme (at the left of Figure 1), there is knowledge that relates only to the machine (mainly this is the hardware of the calculator or computer): for example, how to change the batteries, adjust the screen contrast, reset to factory defaults, access the help system. At the other extreme (at the right of Figure 1) there is the vast realm of mathematical knowledge which is largely unaffected by the presence or absence of technology. In between, however, there is a substantial body of knowledge involving both mathematics and the machine and it is this body of knowledge and skills that is especially of interest in this paper. We will call it the technical aspect of effective use of CAS. Some examples of the technical aspect, which will be discussed in more detail later, are indicated in Figure 1, and they are distributed along the continuum to indicate (very approximately) the balance between knowledge of mathematics and knowledge of the machine that is involved in each. To help clarify what we mean by 
the technical aspect, we emphasise in Figure 1 that it is almost entirely knowledge and skills related to the software rather than the hardware of the machine. As further clarification, we offer the observation that the information in a computer or calculator manual is usually about the machine-specific knowledge and technical knowledge, but not purely mathematical knowledge. Knowledge and skills at all positions on the continuum are essential for doing mathematics well with CAS. One can no more use a calculator with an unreadable screen (machine extreme) than one can solve a differential equation without understanding the importance of boundary conditions (mathematical extreme) or without being able to interpret the output from the machine in familiar mathematical notation (a technical aspect). Our focus on the technical aspect is therefore not because it is more essential to using CAS than other knowledge and skills, but because it is of new importance for teachers. (We would also like to remark that the interface between machine and mathematics provides a privileged place for observing and analysing phenomena which are very interesting for research on learning processes in computer environments.)

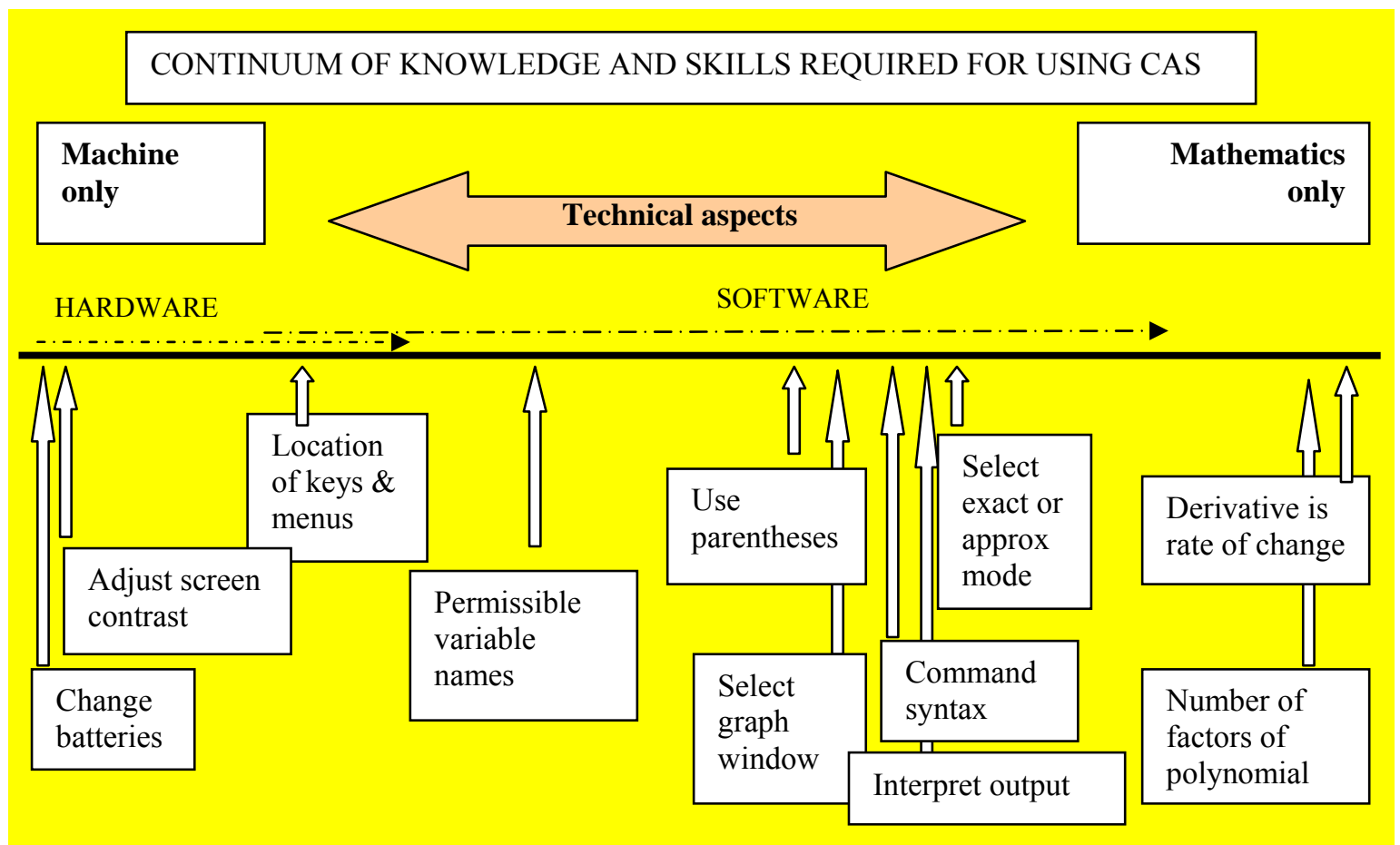


Figure 1. The continuum of knowledge and skills required for using CAS

The above discussion has identified four major aspects (three cognitive and one affective) that contribute to how well a student uses CAS for learning and doing mathematics:

- Mathematical aspect

- Technical aspect

- Machine aspect

- Personal aspect

Incorporating CAS in mathematics teaching requires attention to all of these aspects. Although it provides organisational challenges for teaching, the machine aspect seems to us to be uninteresting pedagogically and is therefore discussed no further in this paper. Exploring the mathematical aspect is of great importance, as coming to fully incorporate using CAS in teaching and learning requires change in mathematical practices. Firstly, there is a need or opportunity to adjust the content of the intended curriculum (see, for example, Kutzler, 1999). Secondly, as explored by Lagrange (1999b), there is a need to conceptualise new practices such as joint development of by-hand and by-CAS procedures. Thirdly, there is a need to change the mathematical emphasis of some teaching, and in the case of CAS, it is particularly in the realm of 'symbol sense' (Arcavi, 1994). In a previous paper (Pierce and Stacey, 2002), we have explored the components of symbol sense that are needed for using a CAS to do algebra, and presented them as a framework for Algebraic Insight to help teachers focus on the new emphases required in teaching.

The remainder of this paper leaves aside consideration of the mathematical aspect and the machine aspect, and offers a framework to analyse the technical and personal aspects of effective use of CAS. We hope that together the frameworks for Algebraic 
Insight and Effective Use of CAS may provide a guide for systematically examining different facets of students' learning with CAS. They should also provide a basis for planning and reflecting on teaching content and practice. They are designed for use by researchers and teachers, to structure observations about students' progress and to identify features of teaching which contribute to students' progress. Whilst the specific details which lead to student difficulties will vary from one CAS to another, because all CAS have their own strengths and weaknesses, our illustration using one particular CAS (the Texas Instruments TI-89) should not imply that this CAS presents more or less difficulties than others.

\section{A FRAMEWORK FOR THE EFFECTIVE USE OF CAS}

\section{INTRODUCTION TO THE EFFECTIVE USE OF CAS FRAMEWORK}

The aim of this framework, as set out in Table 1, is to highlight the technical and personal aspects of CAS use that contribute to how effectively a student is able to do mathematics with this technology. In order to demonstrate the scope of each of the technical and personal aspects, they are subdivided into elements. These elements (column 2 of Table 1) contribute to a student's success using CAS regardless of the particular hardware or software used, the level of mathematics being studied, and the broad context or purpose of the use. This generality does not hold at the level of the next subdivision, the common instances in column 3. The common instances listed are not an exhaustive catalogue of items by which effective use of CAS should be measured but are selected based on the experience of teaching an introductory functions and calculus course with CAS. While the framework does encompass the use of the symbolic, numeric and graphical modules of CAS, the examples of common instances presented here emphasise the symbolic module. This is because in 
the setting where the framework was used, teaching using the graphical and numerical modules was well established, and it was the addition of the symbolic facility that was of current interest. We would like to emphasise that the purpose of the framework is not to provide a list of categories such that each item observed will relate to one, and only one category. This may be desirable but does not seem possible.

\section{TABLE 1}

Framework for the Effective Use of CAS

\begin{tabular}{|c|c|c|c|c|}
\hline \multirow{2}{*}{$\begin{array}{l}\text { Aspects } \\
\text { 1. Technical }\end{array}$} & \multicolumn{2}{|c|}{ Elements } & \multicolumn{2}{|c|}{ Common Instances } \\
\hline & 1.1 & Fluent use of program & 1.1 .1 & Enter syntax correctly \\
\hline & & syntax & 1.1 .2 & Use a sequence of commands and \\
\hline & & & & menus proficiently \\
\hline & & & 1.2 .1 & CAS plot a graph from a rule and \\
\hline & 1.2 & Ability to & & vice versa \\
\hline & & systematically change & 1.2 .2 & CAS plot a graph from a table and \\
\hline & & representation. & & vice versa \\
\hline & & & 1.2 .3 & Create table from a rule or vice \\
\hline & & & & versa \\
\hline & & & 1.3 .1 & Locate required results \\
\hline & 1.3 & Ability to interpret & 1.3 .2 & Interpret symbolic CAS output as \\
\hline & & CAS output & & conventional mathematics \\
\hline & & & 1.3 .3 & Sketch graphs from CAS plots \\
\hline & & & 1.3 .4 & Interpret CAS non response \\
\hline \multirow[t]{4}{*}{ 2. Personal } & 2.1 & Positive attitude & 2.1 .1 & Value CAS availability for doing \\
\hline & & & & mathematics \\
\hline & & & 2.1 .2 & Value CAS availability for learning \\
\hline & & & & mathematics \\
\hline
\end{tabular}




\section{THE TECHNICAL ASPECT: WHERE MATHEMATICS MEETS MACHINE}

As described above, the technical aspect relates to the region where mathematical knowledge and machine knowledge (of the hardware and software) most interact, as depicted diagrammatically in Figure 1. The technical aspect relates to students' ability to access the capabilities of their own CAS to achieve mathematical goals. One welltheorised approach which explicates the need for teachers to attend to the technical aspect, is that of Guin and Trouche (1999). They point out the existence of a complex process, which they call 'instrumental genesis' (following Verillon and Rabardel, 1995), which is required to turn the CAS machine into a 'mathematical instrument' which a student can use skilfully. Such a process must be supported by the active involvement of the teacher employing carefully-designed problem situations. Researchers, including Atkins et al. (1995) and Lagrange (1999a), have pointed out that learning to use the technology of a CAS presents new, additional challenges for students, although it is worth remarking that the students described in Pierce (1999a) mostly felt impeded in their mathematical work only when the CAS was new to them.

Guin and Trouche (1999) analyse the specific features of doing mathematics with CAS by studying the constraints inherent in CAS and the resources that it affords for action by students and teachers. Resources for action include the opportunity to promote experimental work, new ways to understand concepts, and the availability of multiple representations of functions (with the associated need for new skills such as "window shopping" (Hillel, 1993)). Amongst the constraints they list the apparent 
inconsistency and limitations of CAS (so that a CAS may use an exact value for $\cos (\pi / 8)$ but not $\cos (\pi / 16)$, or that it will simplify some complex expressions but not other similar ones), constraints of graphing due to the finite number of pixels on the screen (discretisation), the demand for strictness of some syntax, and the connections between exact and approximate solutions. They also observe that although these constraints cause difficulties for students, they can often be used as a focus for training. Guin and Trouche have demonstrated the importance in attending to the technical aspect of the effective use of CAS (and thus its great interest for the researcher). The overhead involved in becoming a competent user will vary from CAS to CAS but technical facility is essential for a student eventually to be able to focus on the mathematics rather than the machine.

For the technical aspect, the elements in our framework reflect the stages of a student's CAS use: entering expressions using command syntax, then using command sequences for working within one representation (in this case, the symbolic representation) (1.1), navigating between the modules to change representations where appropriate (1.2), and reading and interpreting CAS output (1.3).

\section{Technical element 1.1: Fluent Use of Program Syntax}

Fluent Use of Program Syntax entails the translation of conventional mathematical syntax to CAS syntax and knowing which buttons to push, or which commands, to execute it. Common instances of fluent use of program syntax are seen when students enter syntax correctly, or use a sequence of commands and menus proficiently. Because of the focus that we have chosen on the new aspects of CAS for teachers, the common instances that we discuss here are centred on the symbolic features of CAS.

Guin and Trouche (1999) comment that the syntactic requirements of CAS can be demanding and have to be memorised. Students who took part in Lagrange's study 
(1999a) generally thought that they had used CAS quite easily but when they were tested on their use of basic commands it became clear that their knowledge was quite limited. An early example of students' difficulty with program syntax, and hence something that teachers need to attend to at the start, concerns the rules about implicit/explicit multiplication. Most CAS require a multiplication operation to be explicitly inserted between algebraic symbols, sometimes or always. One consequence of this is that multi-character variable names, such as "speed", that are discouraged in by-hand algebra are permissible in many CAS and students can frequently use these to advantage to keep track of the meaning of expressions.

Other examples of the framework element 1.1.1 (enter syntax correctly) require mathematical knowledge to analyse the structure of the expression to be entered. The first author (Pierce, 1999a) observed that while students showed no problems adapting to the use of CAS symbols such as / for division, ${ }^{*}$ for multiply and ${ }^{\wedge}$ for raise to a power, they commonly had difficulty with the use of parentheses. This difficulty was seen even when the syntax required no more than writing a conventional mathematics expression on one line, for example $\frac{x^{2}+5 x+6}{x-2}$ as $\left(x^{\wedge} 2+5 x+6\right) /(x-2)$. Lagrange (1999a) also comments that the use of parentheses was a common source of difficulty for the students he observed. Using parentheses can be further complicated by differences between by-hand and CAS conventions. For example, the Casio FX2.0 CAS calculator sometimes accepts syntax where parentheses are left un-closed and some students like to use this time-saving, but counter-mathematical, feature.

Other use of syntax requires deeper understanding. For example, it is sometimes quite difficult for students solving a set of equations to specify which variable is to be found in terms of which unknowns, whereas a by-hand solution would proceed more or less by default. These examples of the common instance "enter syntax correctly" 
demonstrate how the technical aspect of effective use of CAS involves both mathematical and machine knowledge intertwined.

In addition to observing the syntax conventions, students must also learn to appropriately use the modes or settings offered by the software, and again this knowledge is often in the technical aspect because it mixes mathematical and software understanding. A deceptively simple example is seen when students must decide whether to work in 'approximate' or 'exact' mode. The choice must be informed by an understanding of the mathematical differences between exact values and decimal approximations and the possible advantages of each. This is technical knowledge because students need to be aware of the alternative mode settings offered by their CAS, how to access and change these options, the differences in syntax required in each mode, and not least to understand the mathematics needed to make appropriate choices.

A different type of example for technical element 1 is being able to work with defined functions. There is no operative need to define a function in by-hand mathematics. One may need a definition for clarity of records, but not in order to carry out operations. This is quite different with some CAS, where a formal process of defining a function is required before an expression such as $f(x)$ or $f(2)$ can be used. Other complications related to element 1.1 of the framework arise for some CAS when a function is needed in both the graphical and symbolic modules of a CAS, which may not share information.

\section{Technical element 1.2: Ability to Systematically Change Representation}

Whereas the element 1.1 in the framework relates to work within one module of the software, this element involves moving between modules. In the case of teaching mathematics with CAS this especially involves the ability to systematically change 
representation. This ability is demonstrated when students use CAS to, for example, plot a graph from a given rule or plot a graph from a table. The ability to move quickly between algebraic, graphical and tabular representations of functions is often said to be one of the strengths of CAS, but Lagrange (1999b) and others have noted that swapping representations can be a source of difficulty. Students need to develop a mental map of the architecture of the system, to understand how the symbolic, graphical, table and home modules are connected and not connected. Figure 2 shows the nature of the sequences required to move between representations when using common CAS calculators. For example, if a function has been entered into the symbolic module, it may need to be transferred to the graphical module (and the variables changed to $\mathrm{x}$ and $\mathrm{y}$ ) before it can be graphed. The results for regression procedures, which find rules to 'fit' tabular data, are commonly stored in the graph module's function list and then must be copied to the symbolic module for manipulation. Hence there is no direct arrow between the tabular and symbolic modules in Figure 2. Such CAS specific routines will require practice to become automatic and so not interrupt students' mathematical thinking. 


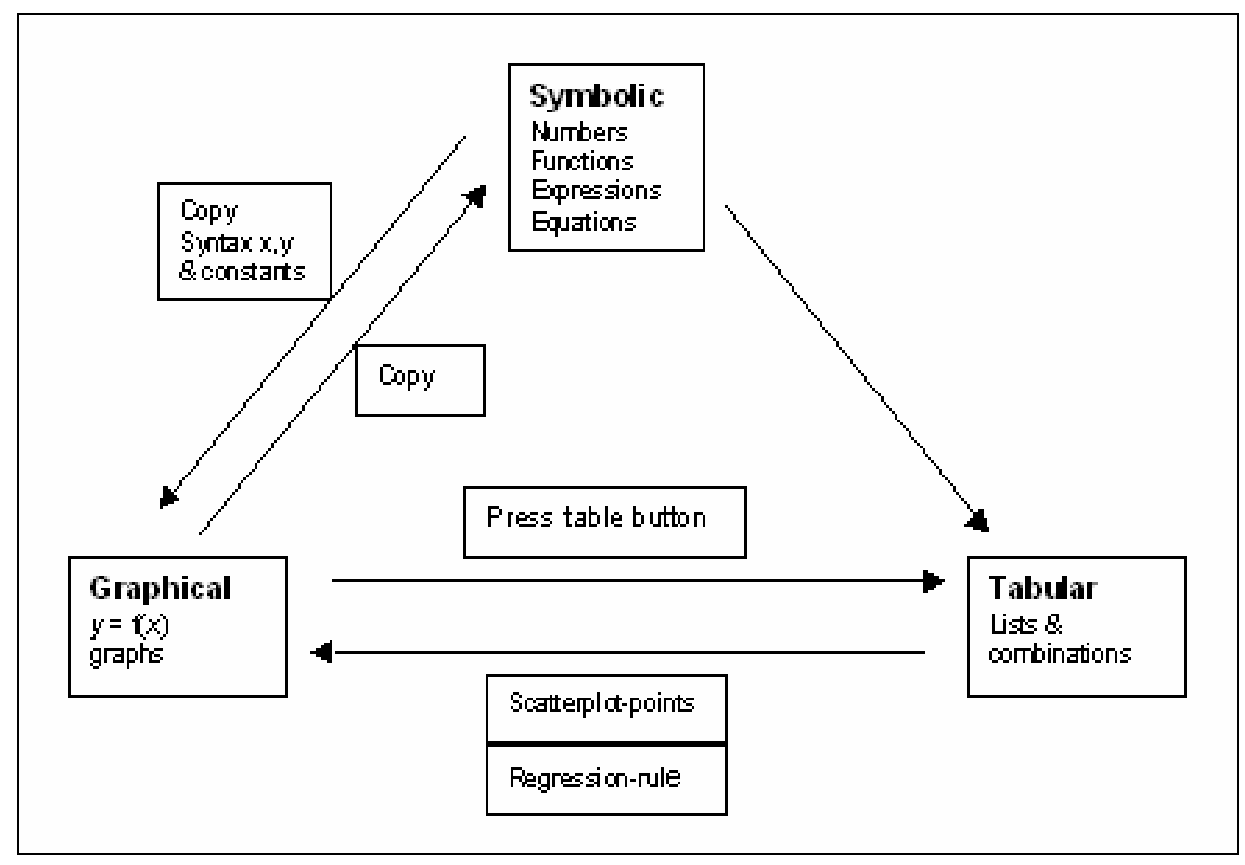

Figure 2. $\quad$ Processes for changing representation modules in CAS

\section{Technical Element 1.3: Interpreting CAS Output}

The third element of the technical aspect, Interpreting CAS Output, can cause significant difficulties which lessen the degree to which students make effective use of CAS. Students must interpret the information on the CAS screen in terms of conventional mathematics. 'Interpret' here refers to the specific understanding needed when the format of results is different from the conventional 'pen and paper' presentation, or is constrained by the limitations of the screen or when the results are unexpected due to unappreciated aspects of the software; it does not include the substantial mathematical task of deriving meaning from the results in terms of the original problem or a real world interpretation or strictly mathematical understanding such as knowing that $i$ relates to complex numbers. Thus our emphasis here is on interpretation issues related to the CAS hardware and software, rather than the mathematical ideas themselves.

Drijvers (2000) observes that one obstacle for students learning CAS is the fact that CAS solutions may be presented differently from by-hand solutions. The first 
author has observed this in the confusion that arose from the CAS presentation of negative indices for expressions that students would normally write as quotients (Pierce, 1999a). Another example is where students are confused by CAS solutions to quadratic equations, which may be expressed in a different way from the conventional form achieved by using the quadratic formula by hand (eg. $\frac{-5 \pm \sqrt{105}}{4}$ ). CAS may give two separate solutions underneath each other or across the screen, which requires scrolling to view the second solution. In either case the student must know how to view 'large' expressions. In addition to this machine skill, the student also needs algebraic insight (Pierce \& Stacey, 2002) to expect multiple solutions and to recognise equivalent expressions when comparing CAS output with conventional byhand formats. This example shows how machine and mathematical knowledge work together in the technical aspect.

A different type of common instance of difficulty interpreting CAS output is seen when, after a command such as solve or factorise, the CAS returns an identical expression or 'no solution'. Interpreting such responses, which students tend to regard as 'non-intuitive', requires both algebraic insight and an understanding of the conventions and limitations of the technology being used. A simple example is when students divide 4 by 5 and receive the response $4 / 5$ in exact mode, instead of the 0.8 that a scientific calculator would produce; some of our students first believed that the CAS calculator had not "worked". In example A, shown in Figure 3, the first factor command returns the original cubic since it works in exact arithmetic, whilst the second factor command, differing only in a detail of syntax, factorises over the real numbers in approximate mode. The student needs the mathematical knowledge to expect that there will be real factors, as well as knowledge of the machine-specific 
alternative syntax. In example B, Figure 3, the CAS response 'false' indicates that there are no real solutions, although a different command outputs complex solutions.

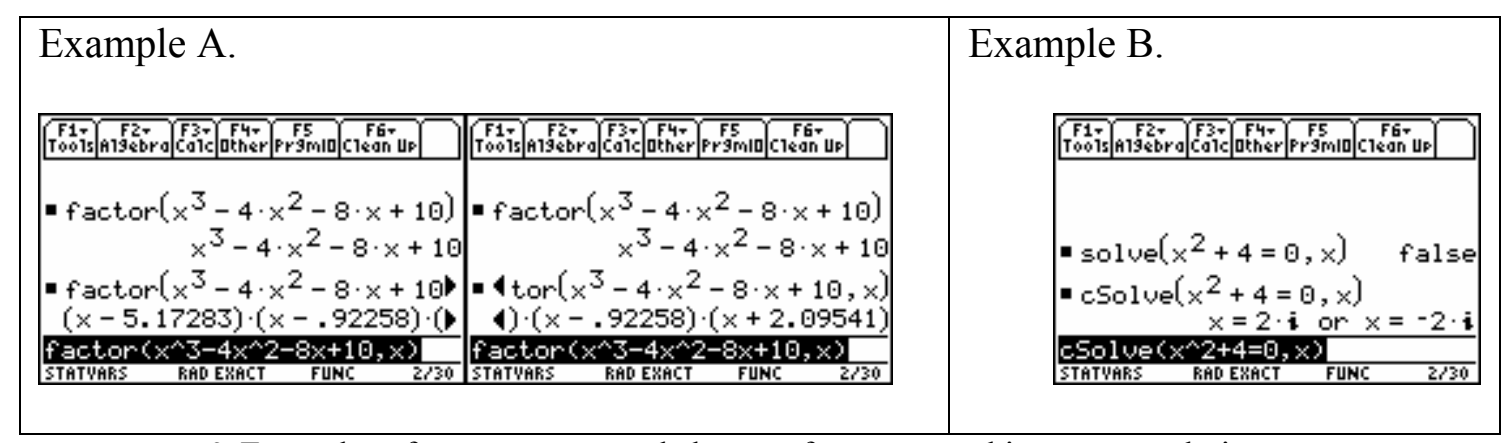

Figure 3 Examples of non-response and change of syntax to achieve some solutions

In each representation (symbolic, numerical or graphical), the design of the software combined with the physical limitations of the hardware can result in unfamiliar messages or formats which may make students' use of CAS less effective. As CAS programs evolve, with, for example, interfaces becoming closer to the format of conventional mathematics, and as screen technology improves, these technical difficulties of interpretation are likely to diminish. However, even if there were few technical difficulties using CAS, the second aspect, the personal aspect would still impact on how effectively students use CAS.

\section{THE PERSONAL ASPECT}

Our observations of students, some of which are documented below, lead to the identification of two different elements of the personal aspect. Element 2.1 concerns having a positive attitude towards using CAS for learning and doing mathematics. This however is not enough. It is also important that a student's manner of use is conducive to good learning and to establishing approved and efficient mathematical practices. This is the basis of element 2.2, judicious use of CAS. 


\section{Personal Element 2.1: Positive Attitude}

The first personal element is a positive attitude towards the use of CAS. This can be seen when students value the availability of CAS as a tool for both doing and learning mathematics. Students with a positive attitude can be expected to be more persistent with CAS use to overcome initial technical difficulties. Students with a negative attitude towards the use of CAS may be less persistent, and more likely to avoid its use. (This conjecture is supported in the case studies described below.)

Students who, as a result of their previous experiences, believe that 'computation equals mathematics' may feel that the use of CAS is illegitimate (Pierce and Stacey, 2001b). The strength of the symbolic facility of a CAS is its consistent implementation of algorithmic processes: what it does best is compute. Students who enjoy doing algorithmic computation and are good at it, but who do not appreciate the broader scope of mathematics, may feel that having CAS devalues their skills. They may be unaware that work with CAS requires a broad range of types of mathematical thinking. Arnold (1995) reports that students who viewed mathematics as 'answerbased' dismissed the possibilities for exploration of mathematical ideas that CAS provided. They valued unaided individual effort and devalued the use of technology. Lagrange (1996) also comments that not all students want to be relieved of pen and paper work. However, a positive attitude towards CAS does not require a correspondingly negative attitude towards by-hand methods, or any other technology, but rather an affirmation of the place of CAS in the range of tools available for doing and learning mathematics.

Most students, as reported in Pierce and Stacey (2001b), agreed that CAS is a helpful tool for producing answers to mathematics problems. We describe this as functional use and suggest through the framework that it is worthwhile monitoring 
this as a common instance (2.1.1) of the element of positive attitude. When asked whether they felt the machine actually 'did' the mathematics, students could see roles for both themselves and for the technology. This is reflected in responses such as:

Student C: I reckon that we are actually doing it [the mathematics]. The computer only spits out an answer to what you type into it.

Student D: You still have to interpret the answer or for that matter interpret the question so you can convert it into what the computer wants...you're still doing a lot of mathematics.

In a similar way, students show evidence of positive attitude when they value the availability of CAS for pedagogical use (common instance 2.1.2), i.e. to facilitate exploration of concepts and so support their learning of mathematics. Pierce (1999a) reports that most students agreed that the use of CAS aids learning, nevertheless many students felt that CAS should only be used after they have learned to do the procedures by hand. When questioned, these students said that they believed mathematical understanding would be shown if they could do the mathematical manipulation by hand and without help. This observation suggests that a student's attitude to the use of CAS for learning and doing mathematics is affected by their beliefs about what constitutes valid learning of mathematics.

It is evident that a student's attitude towards CAS will affect their level of engagement with the technology. There is a clear role here for the teacher in helping students think about these issues; we will return to this below when describing teaching strategies linked to the framework.

\section{Personal Element 2.2: Judicious Use of CAS}

Guin and Trouche (1999) have observed students using various avoidance strategies, which result in various 'unthinking' forms of CAS use. For example, automatic translations of questions in terms of commands, translating a problem 
statement 'word for word' without close analysis of the meaning; over-generalising the applicability of commands (e.g. on the TI-92, using solve for inequations as well as equations); random trials and 'zapping' quickly between commands on the same menu. These behaviours are all negative instances of the second element of the personal aspect, which we call Judicious Use of CAS.

Judicious use is seen when a student implements a reasoned plan for their CAS use to solve a problem or to increase their understanding of a mathematical concept. The first common instance of judicious use is the manner in which CAS is used (2.2.1: Use CAS in a strategic manner). Whilst there can be links between attitude and manner of use, these are distinct elements in the framework (cf. Guin and Trouche, 1999). Following the work of Arnold (1995), we have classified the manner in which students are disposed to use CAS as: passive (just observing others); random (trying anything); responsive (using CAS according to directions); or strategic (following their own reasoned plan). Strategic use is the most desirable and hence the common instance (2.2.1) is named after this. In the authors' experience, when questioned about their approach to using CAS, one student may say "Oh I just did one of everything [on the menu]" while a second may say "I chose these graphs because they show...". These comments suggest that the first student is using CAS in a random manner while the second takes a strategic approach.

Guin and Trouche (1999) categorised what they called 'work methods'. They used the terms random, mechanical, resourceful, rational and theoretical. In the example above the term 'rational' could be applied instead of 'strategic'. For either classification, as Guin and Trouche explain, "the categories are not exclusive but useful for identifying extreme types of behaviour and describing change over time" (p. 215). 
The second common instance of judicious use (2.2.2) concerns the choices students make about when to use CAS. We value students being discriminating in their functional use of CAS; that is, they choose to use CAS for lengthy computation, but choose a by-hand solution for a quick, straightforward calculation. Such behaviour requires a disposition to plan ahead, considering the nature of the task, in addition to mathematical and technical skills.

The final common instance of judicious use (2.2.3) is evident when students, who will almost certainly have an attitude of valuing the use of CAS (2.1.1 and 2.1.2), undertake pedagogical use of CAS to help them learn a new mathematical concept or procedure, or to gain a broader or deeper understanding of familiar mathematics. For example, a student may explore a family of functions in order to understand the effect of varying some parameter, using CAS to look at multiple examples and some nonexamples of that family of functions. This can also involve using multiple representations of functions in order to illustrate a concept in a number of ways. The same common instance can be seen where teachers become more sophisticated and skilled in teaching with technology such that there is often a transition from viewing CAS as a mathematical assistant primarily for functional use, to viewing CAS as a tool for learning; Pierce and Stacey (2001a) document one such transition of this kind.

This section has described the key aspects of the framework for effective use of CAS. The number of framework elements has been kept to a minimum, to make the framework easy to remember and to use. The next sections illustrate how the framework might be used, firstly for monitoring students' progress and secondly to organise ideas for teaching. 


\section{USING THE FRAMEWORK TO MONITOR STUDENTS’ PROGRESS}

This section illustrates how the framework can be used to monitor student's progress in learning to use CAS effectively and how it can consequently draw attention to issues for teaching. Four case studies are presented, drawn from seven reported in detail in Pierce (2002). The case studies were selected for inclusion here because each represented a different combination of self-reported general computing skills and calculus background (measured by success in previous school mathematics). Thus the four cases possess varying prior machine-only skills and mathematics-only skills and we report here on the development of their technical skills (where machine skills and mathematics skills must be combined). In order to limit the number of variables in the cases presented, all the cases are female students.

The data were collected over 15 weeks (13 weeks teaching, study week then examinations) when the first author taught an introductory functions and calculus course with a computer-based version of DERIVE to a class of 21 undergraduate students. CAS was made available for all teaching and assessment, and the teacher (the first author) encouraged students to use CAS to explore and find patterns as well as to tackle problems that they would have found difficult to do by hand. In addition, CAS was used for demonstrations and illustrations during lectures. Each week, practical sessions were conducted in a computer laboratory, where students were encouraged to work together using the CAS. Exercises set for the laboratory classes required CAS to be used functionally (to find answers to problems beyond expected by-hand skills) and pedagogically (to explore new mathematical ideas). The textbook used (Weimer, 1998) also encouraged both functional and pedagogical use. The Effective Use of CAS framework was used as a basis for considering whether 
students' CAS use improved during the course, and which students improved in what aspects and why.

Surveys, interviews and observation were used to collect data for overall class results and for detailed case studies. On three occasions (early, mid and late in the course) Technical Difficulties and Judicious Use of CAS surveys were administered to all students at the conclusion of their CAS laboratory session. Students were asked to reflect on their use of CAS during that session and to respond to a series of statements. The statements related to technical difficulties which the teacher knew, from previous experience, were commonly encountered by students when implementing their mathematical decisions (see Table 2). The respondents had the option of 'not applicable' as well as a 5-point frequency scale from 'never' to 'every time'. Further statements relating to judicious use of CAS (see Table 3) were presented in a single multiple-response item. The pattern of responses was used to make judgements about the personal aspect. For example, a pattern of negative responses to statements 1,7 and 8 of this item was taken as evidence of a negative attitude. The early-course survey also contained extra questions concerning students' prior use of technology for mathematics and the late-course survey included questions on when students chose to use CAS and if they found CAS helpful.

Observations of CAS use were made during laboratory sessions and during examinations. Sometimes assisted by a colleague, the first author as teacher/researcher made field notes, noting the way students were using the CAS and the nature of the questions they asked. The aim of these observations was to both validate and supplement the students' survey responses relating to technical difficulties (framework elements 1.1, 1.2, 1.3) and judicious use of CAS (2.2), as well as providing information about attitude to CAS use (2.1). Volunteer students (which 
included all the case study students) were formally interviewed at the end of the course individually or in pairs by the first author. The interviews enabled students to expand on their survey responses, to react to the teachers' observations of their learning behaviours, and to explain their attitudes to using CAS.

TABLE 2: Statements in the Technical Difficulties Survey, with links to the Effective Use of CAS framework.

\section{Possible area of difficulty}

1. Authoring, using $/,^{\wedge}, *,+,-$ symbols

2. Using brackets to force the structure of expressions

3. Using syntax for commands eg FIT, VECTOR, F(x)

4. Using sequences of commands eg to substitute

5. Interpreting the results of the soLve command

6. Obtaining exact or approximate solutions

7. Working from the screen to ordinary maths symbols

8. Moving to a graph window

9. Obtaining a window which shows the graph required

10. Zooming to show key features of a graph

11. Copying from the screen for sketch graphs

12. Working out the scale of a graph

\section{Framework Element}

1.1

1.1

$1.1,1.2$

1.1

1.3

1.1

1.3

1.2

1.2

1.2

1.3

1.3

TABLE 3: Judicious Use of CAS survey items, with links to the Effective Use of CAS framework

Please tick as many of the statements below as apply.

Today I have used DERIVE to: 


\begin{tabular}{ll}
\hline Find answers if computer use was suggested. & 2.2 .3 \\
Explore problems if computer use was suggested & 2.2 .3 \\
Explore variations on the set problems. & 2.2 .3 \\
Explore other than when directed, but on the same topic & 2.2 .3 \\
Explore other mathematics not on today's topic & 2.2 .2 \\
Find answers I could 'easily' have done with pen and paper. & 2.2 .2 \\
Find answers to 'hard' questions & 2.2 .2 \\
Find answers to time consuming questions & \\
\hline
\end{tabular}

\section{A GLOBAL PICTURE OF THE CHANGES OBSERVED IN THE STUDENTS}

In Pierce (2002), the data from the various sources are cross-validated and assembled into detailed profiles for all the students, matched against each of the framework elements. The class, as a whole, came to use CAS more effectively, but it was clear that not all students responded in the same way. Students experienced different levels of technical difficulty and there were important individual differences in both students' attitudes toward the use of CAS and their judicious use of CAS.

For the purpose of giving an overview across the framework elements, the data have been combined to provide a global indication of change during the course. Figure 4 shows how the four students changed during the course using four gradations for each framework element. For the three technical elements $(1.1,1.2$ and 1.3) the shading from dark to light indicates very many to very few technical difficulties reported (in comparison to the class average). For element 2.1 (positive attitude), the shading indicates very low (dark) to very high (light) value placed on having CAS available for doing and learning mathematics. For element 2.2, the shading indicates the extent of judicious use from very dark (passive or random use, not discriminating and not used pedagogically) to very light (strategic and discriminating functional use 
and appreciating pedagogical use). Figure 4 assists us to review the progress of four individuals by summarising the data obtained from class observations, worksheets, interviews and examinations, which are reported in more detail below.

\section{Effective use of CAS: student progress chart}

\begin{tabular}{|c|c|c|c|c|c|c|c|c|c|c|c|c|c|}
\hline \multirow[b]{2}{*}{ Aspect } & \multirow[b]{2}{*}{ Element } & \multicolumn{3}{|c|}{ Jennifer } & \multicolumn{3}{|c|}{ Louise } & \multicolumn{3}{|c|}{ Yvonne } & \multicolumn{3}{|c|}{ Jocelyn } \\
\hline & & $\mathrm{E}$ & $M$ & $\mathrm{~L}$ & $\mathrm{E}$ & $M$ & $\mathrm{~L}$ & $\mathrm{E}$ & $M$ & $\mathrm{~L}$ & $E$ & $M$ & $\mathrm{~L}$ \\
\hline \multirow{3}{*}{1 Technical } & 1.1 & & & & & & & & & & & & \\
\hline & 1.2 & & & & & & & & & & & & \\
\hline & 1.3 & & & & & & & & & & & & \\
\hline \multirow[t]{2}{*}{2 Personal } & 2.1 & & & & & & & & & & & & \\
\hline & 2.2 & & & & & & & & & & & & \\
\hline
\end{tabular}

Figure 4. Chart showing the progress of four students, in terms of the framework elements, from Early (E) to Mid (M) to Late (L) course. (Four grades of shading explained in text)

\section{CASE STUDY 1: JENNIFER}

Jennifer was selected because, relative to others in the class, her prior background in calculus was limited (she studied a low-level year 12 mathematics course at school, which included no calculus and only limited algebra) and she judged herself as having limited general computing skills. She had used a scientific calculator but neither a graphical calculator nor a computer for mathematics. Jennifer was an organised, conscientious student. Throughout the course, observations suggested that Jennifer had a positive attitude to trying to use CAS for learning mathematics (see Figure 4).

\section{Technical}

On the early-course survey, Jennifer reported no problems using the CAS symbols $/, \wedge$ and $*$ in simple expressions or interpreting the results of the solve command but 
did have some problems with other syntax (1.1), menu navigation, working in different representations (1.2) locating solutions and working from the screen to 'ordinary' mathematics symbols (1.3). The researcher's observation notes confirmed that Jennifer had difficulty with syntax for commands, in particular the need to make the structure of expressions explicit through the use of parentheses. While identifying the structure of an expression and hence inserting appropriate parentheses requires mathematical understanding, realising that parentheses are necessary in order for the software to parse the expression also requires technical understanding. Jennifer's difficulties in these areas are properly described as technical because even when she had made appropriate mathematical decisions, she was often unable to operate the CAS in the way she desired.

In addition Jennifer had some difficulties linking representations (graphical and symbolic) due to an inability to choose or set an appropriate scale for the viewing window. There was a strong 'ah-ha' reaction when an appropriately-scaled graph was demonstrated to her. While Jennifer had trouble setting useful scales for graphs her observations from the screen and translation to sketch graphs were excellent.

In the mid-course Jennifer again reported many technical difficulties. While she had no difficulty using standard CAS symbols she encountered problems with complicated expressions that required parentheses, with syntax and sequences of commands, with interpreting graph scale settings and zooming in a controlled manner to see key features, and interpreting screen output as conventional mathematics.

During the post-course examination the observer noted that Jennifer experienced very few technical difficulties Even so, on the late-course survey Jennifer reported that she was still finding some technical difficulties, especially with syntax and graph windows. 


\section{Personal}

Initially Jennifer was uncertain about the use of CAS but she was prepared to keep trying (2.1) and quickly gained confidence despite her relatively severe technical difficulties. From the beginning she embraced the use of CAS for both functional and pedagogical purposes. She reported non-discriminating use of CAS, choosing to use it to find answers to easy, hard or time-consuming questions. At this stage she undertook limited pedagogical use of CAS, choosing to use its facilities to explore variations on the set topic (without direction from the teacher). (This is indicated in Figure 4 by the 2.2 entry showing medium dark shading.)

By mid-course Jennifer had developed a positive attitude and made extensive use of CAS. She used it functionally but was still not discriminating in her approach. Despite her technical difficulties, Jennifer now undertook extended pedagogical use of CAS exploring patterns when this was suggested and took the initiative to extend this beyond suggested variations of parameters.

Jennifer's judicious use of CAS improved throughout the course (as indicated for 2.2 in Figure 4). By late in the course she was making discriminating use of CAS and undertook extended pedagogical use. Her survey responses indicated that CAS had helped her to see patterns and to understand mathematics. She had used it to try out ideas and her confidence with functions, graphs and calculus had improved. In the post-course interview Jennifer said that she had found CAS hard to use at first. She had difficulty remembering the names of the various commands and translating conventional mathematics to CAS, but she especially liked the graphing facility of CAS. She liked to use pen and paper for questions she found easy but preferred CAS for 'hard' questions. Overall she said CAS was 'good' for doing and learning mathematics. 
Jennifer - an overview

Consistent with her low self-rating of computing skills and mathematics background, at each stage of the course Jennifer's level of technical difficulty was relatively high. However she valued the access and support which CAS provided for her learning of mathematics. By mid-course, as the mathematics became more familiar and she gained experience in using CAS, her level of difficulty decreased but it increased again later with new mathematical material. Monitoring Jennifer's progress in overcoming technical difficulties alerted the teacher to the need to assist Jennifer by emphasising the requirements of CAS syntax, providing additional examples and continued encouragement. As a result, Jennifer's attitude was always positive. She progressed from learning the basics of the CAS program, through nondiscriminating functional use, to undertaking extended pedagogical use of CAS on her own initiative. Sometimes she used CAS in a strategic manner. It seems that Jennifer's positive attitude allowed her to benefit from the use of CAS despite the difficulties she had learning to use it.

\section{CASE STUDY 2: LOUISE}

Louise's prior background in calculus was relatively good but, like Jennifer, she judged herself as having limited general computing skills. She had studied middlelevel mathematics at year 12 and had used a scientific calculator but never a graphics calculator or computer for mathematics. Louise missed a few classes, but completed all the set work. Although she lacked confidence in mathematics she was happy to ask questions. In the later part of the course Louise became distracted and her social life dominated her conversations. However, her attitude towards the use of CAS was positive throughout the course. 


\section{Technical}

On the early-course survey Louise indicated that she had some problems entering sequences of commands and complicated syntax; difficulty setting graph windows and making sketches from the 'pixelated' graphs on the screen.

By mid-course Louise encountered few technical difficulties except interpreting output from the screen (1.3). Observations confirmed that Louise was confident in her technical use of CAS. Louise's solutions to worksheet problems suggested that she was able to focus, when she wished, on the mathematics rather than the technology and as a result was starting to see patterns, to look for structure and key features. Unfortunately Louise did not always use her skills to advantage as she often approached her work in an unthinking manner. There would be errors on the screen, such as obvious mismatches with the mathematical expression on her page, but she would not notice. Alternatively, she would unthinkingly copy answers exactly as they appeared on the screen rather than reverting to conventional notation. During the latecourse interview Louise commented that she had not found CAS difficult to use:

\footnotetext{
I found using the computer a lot easier than I thought it would be. Once you get used to what different commands are doing and you put the right amount of brackets in, and you got it to stop beeping at you... [a beep alerts the user to a syntax error]
}

\section{Personal}

From the beginning, Louise valued the availability of CAS as a tool that she perceived saved time and effort. Louise placed great trust in the CAS, using it in both functional and pedagogical ways. Louise commonly chose to use graphs to explore patterns and algebra to find answers (eg simplify or solve). She initiated use of CAS to explore variations on set problems and to find answers to problems she saw as being time-consuming. There was evidence that she made discriminating functional 
use and undertook limited pedagogical use of CAS. Louise demonstrated a positive attitude towards the use of CAS for both doing and learning mathematics.

By mid-course Louise indicated that she now used CAS for any or all questions, making extensive use of CAS to explore variations on problems and to try out things for herself. However classroom observation showed that Louise often did not plan strategies but rather would just try things out, in a random manner, to see what happened. Throughout the course it was common to hear Louise say 'I'm not sure what I'm doing but..." Louise had a tendency to try anything, without considering whether it was sensible. For this reason, the ratings on element 2.2 (see Figure 4) are lower than the description above may imply.

The late-course interview transcripts confirm that Louise remained positive about the use of CAS (2.1). She preferred to do 'simple' problems in her head but harder problems and graphs with CAS (2.2.2). She said that CAS was helpful but that 'you need to know your basics'.

\section{Louise - an overview}

Louise had a positive attitude towards the use of CAS, but she tended to use it with blind trust, in an unthinking way. Few of her difficulties were in using the software. Her problems in moving between conventional mathematics and CAS syntax resulted from a lack of 'algebraic expectation' (Pierce and Stacey, 2002). Her confidence in doing mathematics with CAS increased during the course but she still lacked certainty without CAS. While she used CAS to explore variations, this would have been of more advantage to her learning if she had taken a more strategic approach. She did not make conjectures and think about what the answer to a problem, or the shape and position of a graph were likely to be. Louise's overall 
ratings on 2.2 in Figure 4 therefore averages two opposite trends - a willingness to engage in functional and pedagogical CAS use, but not to do so strategically.

\section{CASE STUDY 3: YVONNE}

Yvonne's prior background in calculus was limited (like Jennifer) but she judged herself as having very good general computing skills (like Louise). Yvonne often referred to herself as "dumb at maths" and displayed mathematics anxiety, expecting to have difficulties with each topic, saying "I won't be able to do this". Yvonne had studied low-level mathematics at year 12 and had used no technology beyond a scientific calculator. However, Yvonne was confident about her general computing skills, rating them as very good and this was confirmed by the teacher's observations. Her attitude towards the use of CAS was positive throughout the course (see Figure 4).

\section{Technical}

On the early-course survey, Yvonne indicated that she had few difficulties using DERIVE's built-in commands and menus. However classroom observation notes record that as Yvonne started to use the CAS, she had problems translating conventional mathematics to and from CAS (using parentheses, input syntax, interpreting answers). She rapidly learned to manage the software. Most difficulties she encountered stemmed from her poor algebraic insight and mathematics anxieties. Yvonne was unsure about what the CAS was doing for her and how to interpret the output.

On mid-course and late-course surveys she indicated few problems using CAS and her confidence slowly increased. Her persisting difficulties were with the use of parentheses and setting appropriate graph windows. This was not because she had any problem understanding the requirements of CAS syntax but because she had difficulty 
identifying the structure of expressions and linking key features of symbolic functions to likely critical values for their graphs.

\section{Personal}

On the early course survey Yvonne added a comment saying that she would expect to have problems using syntax. This was not a negative comment about CAS: she was not anxious about operating the CAS but rather this commented reflected her general mathematics anxiety and it matched her comments in class that she was not using CAS because she "didn't know what (mathematics) to do". Unlike other students at the beginning of the course, Yvonne often sought help with the mathematics but seldom asked questions about how to use CAS. Her early use of CAS was functional and non-discriminating.

In class, Yvonne teamed up with a mathematically strong partner and she would either watch him use the CAS or do as he instructed. However, by mid-course, Yvonne was starting to try things out for herself, undertaking pedagogical use (2.2.3). She explored variations of set problems when this was suggested and asked the teacher questions of the form "Is this an example of...?" When she found patterns or made correct conjectures she was very pleased and expressed confidence. Her CAS use (2.2.2) had become discriminating, as she used it to find answers to hard or timeconsuming questions.

By late-course Yvonne would use CAS for any question, even simple items that she could have done in her head (2.2). This was partly due to her mathematics anxiety but also because she liked using CAS. She valued it as an instrument for both doing and learning mathematics. Yvonne felt that using CAS had helped her to see patterns and to understand mathematics and indicated that her confidence had increased in all areas of mathematics studied during the course. 


\section{Yvonne - an overview}

Despite starting with very good computing skills, Yvonne's limited mathematical knowledge meant that she had difficulty using the CAS program at the beginning of the course. She made little use of CAS because she didn't know what she wanted it to do, and she could not make a link to conventional mathematics (1.1 and 1.3).

Working with a partner assisted her learning and she became confident to work on her own, doing mathematics with CAS to overcome the anxiety of unsupported pencil-and paper work. She developed positive learning strategies such as making conjectures, thinking about what she expected an answer or graph to be, and learning from the experience of testing these ideas. She used CAS effectively to follow this style of learning in combination with the use of multiple examples and graphical representations. Her attitude was always positive and, as in indicated in Figure 4, she made progress through the course on each of the other elements of the framework.

\section{CASE STUDY 4: JOCELYN}

Jocelyn had a sound basic background in calculus (having studied a mid-level Year 12 mathematics course including calculus) and she judged herself as having good to very good general computing skills. However, her attitude towards the use of CAS was more negative than other students throughout the course (see Figure 5), so she is an interesting case study. Jocelyn attended class regularly, submitted set work and was confident to ask questions in whole class setting and individually. She had previously used both a scientific and graphical calculator but had not used a computer for mathematics. She was quite confident about her school mathematics ability but reluctant to learn new methods. Her attachment to the graphing calculator, which she still retained, and a reluctance to learn a new technology was to dominate her experience with the new computer-based CAS in the course. 


\section{Technical}

Early in the course Jocelyn said that she had a lot of difficulty thinking of, or remembering, the appropriate sequence of commands to carry out procedures (1.1). She also had some difficulty with entering correct syntax and interpreting exact and approximate solutions (1.3). Within the graphical representation in DERIVE, Jocelyn could not successfully use the menu commands to zoom and focus on a smaller section of the graph (1.2). Jocelyn's difficulties were not due to poor algebraic insight but related directly to her unwillingness to learn the idiosyncrasies of the program.

By mid-course Jocelyn still had difficulty entering expressions, using parentheses correctly in the syntax, changing representations and setting the appropriate scale for graphs and then interpreting CAS output. Only late in the course, as she acknowledged that CAS use would be expected in the examination, did Jocelyn allow the teacher to revisit basic technical skills with her.

\section{Personal}

From the beginning of the course Jocelyn's use of CAS was limited and she did not want to use CAS. She demonstrated non-discriminating functional use and undertook a minimum of required pedagogical use. She said that she used CAS only if a problem explicitly stated 'use CAS to...'. By mid-course Jocelyn still only used CAS when it was suggested but now discriminated and only used it for 'hard' questions, resulting in an improved rating for 2.2 in Figure 4. She said 'I'm still scared of the computer and try to do most [problems] by pen and paper". In the midcourse examination her use of CAS was limited and error-ridden. It was of no advantage to her in answering any question. 
Later there was a small breakthrough when Jocelyn, with initial help from the teacher, used CAS to explore the behaviour of a family of functions and its derivatives. She declared "Oh that was fine. I didn't even need to do them all - I could see the pattern". However Jocelyn did not take the initiative to use CAS to explore mathematics and was not always discriminating in her functional use. On the post-course survey Jocelyn agreed that using CAS helped her to see patterns but she disagreed that it helped her to understand mathematics. She indicated that her use of CAS was no longer restricted to only using it when instructed to do so. In the postcourse general interview Jocelyn said of CAS:

I don't like it. I find it really daunting. I reach for my graphical calculator before it ... It is hard to know what to do, all your Manage, Substitute stuff [sequences of commands and syntax].

Jocelyn - an overview

Jocelyn chose to use CAS as little as possible and so found that it was hard to remember procedures that were not intuitively obvious. She had syntax difficulties throughout the course (1.1 and 1.2) and, while she became marginally more fluent without practice, she remained anxious about using the computer. Jocelyn's case highlights the importance of teachers providing regular practice for students to master CAS procedures. Reluctant users need encouragement and structured practice.

At the same time Jocelyn valued the graphing calculator which she had used in her final secondary school examinations. (Jocelyn's reluctance to use CAS cannot be explained simply by a 'fear of computers'; a transition in the following year to the use of TI-89 calculators rather than DERIVE did not alter Jocelyn's negative attitude towards CAS.) She persisted as a non-user or random user (2.2) throughout the course, preferring pen and paper and the (non-CAS) graphing calculator. Despite her 
dislike of CAS, she was not even consistently discriminating in her use of it. Jocelyn did not make the necessary effort to become confident in her use of CAS and so its use did not improve her algebraic insight. When she did use it she was focusing on 'what to do to run the program' not on the mathematics.

For Jocelyn, her prior school experience largely outweighed the new 'local' institutional value placed on CAS. She valued her graphical calculator which had been allowed in her high-stakes secondary school examination, while CAS was prohibited. Only when she acknowledged that CAS could help in the course examination did Jocelyn begin to engage with the CAS. Generally, to develop institutional value, a teacher can provide a role model, indicating ways in which a CAS can be important. This may be achieved by demonstrating with CAS in lectures or classes, using a view screen or data projector. Students can then see not only how CAS may be used but also that it is OK to make mistakes, how to recover from them, and how to make a positive response to error messages. This can prevent technical difficulties becoming a cause for negative attitude. The case of Jocelyn is a reminder that even careful teaching and enthusiastic modelling is not always able to overcome students' entrenched attitudes.

\section{REFLECTIONS ON USING THE FRAMEWORK FOR MONITORING}

The case studies have demonstrated some of the wide range of ways in which students use CAS and come to learn to operate it effectively. There is a great deal of variation in students' achievement against the framework elements. All four students made overall progress towards effective use of CAS and progressed in most of the framework elements (as did the class overall, see Pierce (2002)). Element 2.1, attitude, seemed very stable with only small changes during the course and this seemed to influence progress in other elements. Technical and personal aspects are 
interwoven and they are also interwoven with mathematical knowledge, although this is not the subject of this paper.

The case studies illustrate that there can be significant variety in students who have similar overall rankings (e.g. in summaries such as Figure 4). For example, judicious use of CAS (element 2.2) appears in these case studies in the form of rather disparate common instances. Louise, for example, demonstrates that students may not make strategic use (2.2.1), but may still appreciate CAS's pedagogical value (2.2.3). Jocelyn was sometimes discriminating (2.2.2) but did not adopt pedagogical use (2.2.3). Yvonne was not discriminating in her functional use but developed a strategic approach to pedagogical use. Jennifer was (eventually) high on all categories. It therefore seems important that the teacher or researcher considers all three common instances for this element: whether use is strategic, whether functional use is discriminating and whether the student appreciates the pedagogical possibilities of exploring mathematics with the technology.

The case studies (and other results in Pierce (2002)) indicate that the three elements of the technical aspect can be expected to develop in different ways. Over the course, students became generally better at fluent use of program syntax (1.1) but as new areas of mathematics were encountered new sequences of commands had to be learned. Technical difficulties lessen but do not disappear. With the exception of Jocelyn, who gave herself very little practice, the students became better at moving between the computer modules (1.2). These skills were required throughout this course, so there was general improvement as skills continued to strengthen. Similarly, skills continued to strengthen in terms of ability to interpret CAS output (1.3) but in this case, rather than the students understanding the limitations and conventions of the CAS, students' mathematical knowledge seemed to play a more important role, so 
that Jocelyn and Louise found this easier than Jennifer and Yvonne, who had weaker mathematical backgrounds.

The case studies illustrate the interdependence of the technical and personal aspects. In planning teaching, attention needs to be given to both of these aspects. Comparing the experiences of Jennifer and Yvonne with that of Jocelyn suggests that students' technical difficulties may be easier for teachers to overcome than personal attitudes and work habits. In addition, Jennifer's case demonstrates that persistent, low level technical difficulties need not prevent the use of CAS as a valuable learning tool.

\section{TEACHING TO PROMOTE THE EFFECTIVE USE OF CAS}

The report of the case studies above has shown how the framework can be used to monitor students' progress towards effective use of CAS, and it introduced the students' reactions to some of the teaching strategies used in the program. This section organises teaching strategies with respect to the framework (see Table 4). Further detailed discussion of the decisions made about teaching strategies and their effectiveness is given by Pierce (2002).

For any mathematical software tool, it is essential to plan to teach specific technical skills: input syntax and command sequences (1.1), navigating between the modules (1.2) and reading and interpreting output (1.3). All the evidence we have considered (in the literature, and from our own research studies) demonstrates that each of these elements can cause difficulty for students learning to use CAS, so each needs attention. Table 4 lists teaching actions that are especially relevant to each element. For developing fluent use of program syntax (1.1), teaching should provide clear 'how to' information and regular practice, introducing features one-by-one as required for course content. In our experience, only a very few students have 
expressed a desire initially to have access to CAS reference manuals: they preferred to be taught command by command, so that they can build their own notes. This repertoire of commands needs to be expanded as new areas of mathematics are introduced. In our research studies, there has always been an increase in technical difficulties when new commands were required, but over time students do adopt new techniques more rapidly. Command sequences that are not intuitive are easily forgotten and when using CAS on a calculator there is currently very little on-line help available. Students like Jocelyn who refused to practice made slow progress in overcoming technical difficulties. The examples in the case studies above have concentrated on the symbolic aspects of CAS, but each representation requires its own program syntax to be carefully taught.

The other technical elements, 1.2 and 1.3, we will not discuss in detail here they are summarised in Table 4.

Addressing the personal aspect of effective use of CAS may be more difficult than equipping students to deal with technical demands. Especially in this time of transition, as we explore new uses of technology in teaching and learning mathematics, both students' and teachers' responses will be strongly influenced by their past experience and the aspects of the new learning which they value, and feel that others value. The case studies suggest that developing positive attitudes (2.1) rests upon using strategies to give CAS institutional value (use by teacher, use in assessment) and careful management by the teacher of the additional burden of overcoming technical and machine difficulties. Similarly, developing judicious use of CAS (2.2) rests upon using classroom tasks where using CAS has clear, genuine pedagogical or functional benefit and undertaking in the classroom shared reflection on strategic and discriminating CAS use. The teacher should discuss with students 
what thinking and tasks they do and what tasks they assign to the CAS and encourage students to critically analyse how they have used CAS in their work. This can be easier in a classroom using computer CAS rather than calculator CAS, where the more private screens make sharing less spontaneous, so that teachers need to more actively engineer discussions (Pierce, 1999b). All these teaching actions are summarised in Table 4.

TABLE 4: Teaching to promote Effective Use of CAS

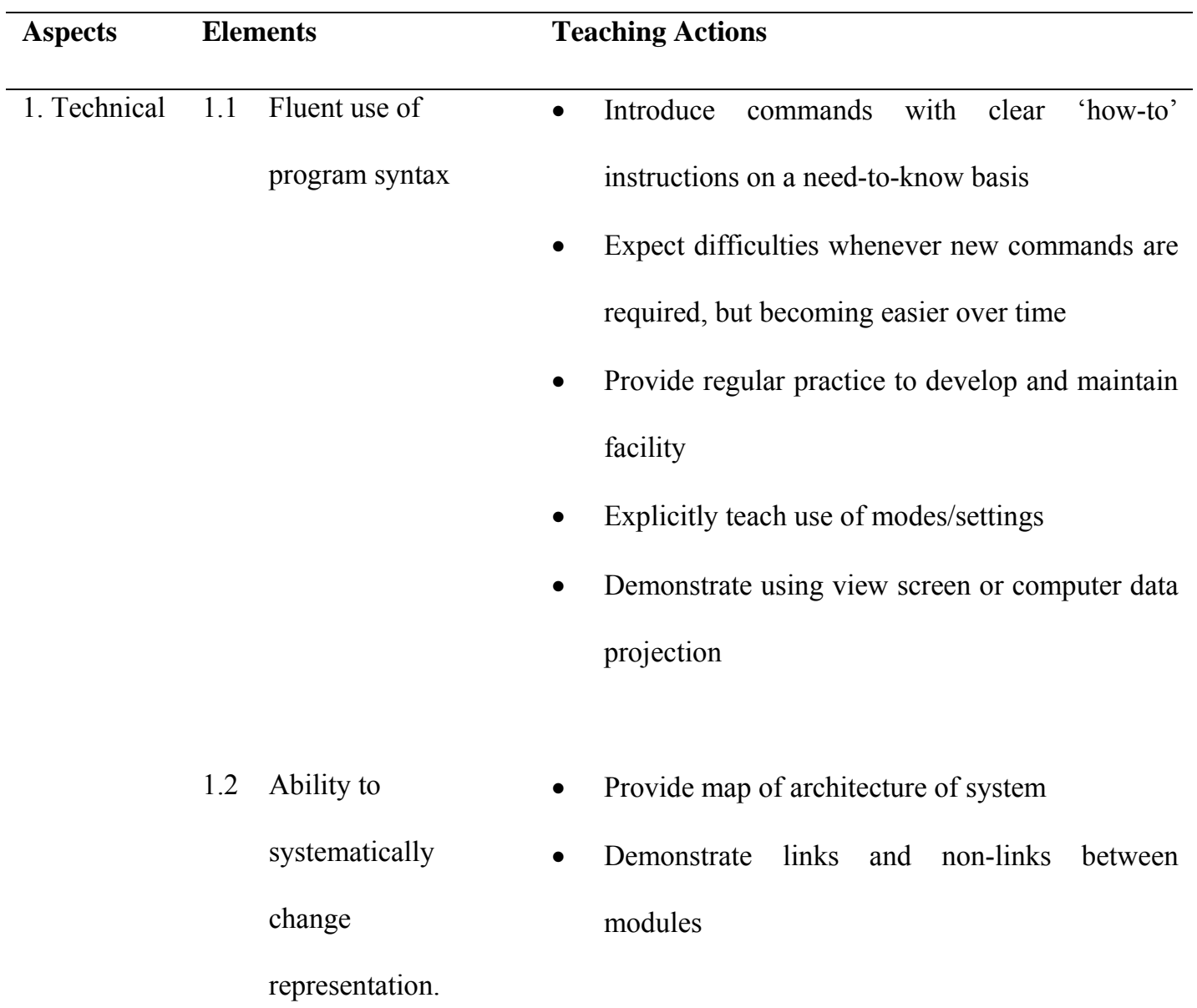


1.3 Ability to interpret CAS output

2. Personal

2.1 Positive attitude

2.2 Judicious Use of CAS
- Discuss format of output (e.g. large expressions, multiple solutions, general solutions)

- Explain error messages, input returned as output, responses such as "false"

- Discuss the effect of different modes

- Teacher uses CAS regularly to demonstrate institutional value

- Allow use in assessment

- Guard against technical difficulties causing negative attitudes

- Foster helpful, sharing classroom atmosphere

- Include questions where CAS really is useful

- $\quad$ Provide questions explicitly requiring pedagogical use (e.g. exploring conjectures)

- Encourage students to monitor their own under/over use of CAS.

- Encourage discussion of strategic by-hand/byCAS balance

- Discuss the contribution of human and machine

\section{CONCLUSION}

The purpose of this paper was to present a framework that outlines the main components that contribute to effective use of CAS, and organises them in a way that is useful for monitoring student progress and planning teaching. Learning to make effective use of CAS involves four aspects of knowledge and skills: mathematical, machine, technical and personal. This paper has concentrated on two of these aspects 
- the personal and the technical (where mathematics meets machine), because these seem the aspects requiring most new teaching attention. The technical and personal aspects have been analysed in terms of a set of elements whose importance has been derived from the research literature. The paper has also demonstrated the framework in action by presenting four case studies of students for whom the effectiveness of their use of CAS has been monitored. Finally, as a result of this monitoring we highlighted in Table 4 matters for special attention in teaching. Here the use of the framework was to provide a structure and checklist.

The framework has been created in the context of the introduction of computer algebra systems into senior school and tertiary mathematics courses, but future work may adapt it to analyse teaching with other mathematical software tools. Some, but not all of the framework, would be affected. The major division into mathematical, machine, personal and technical aspects is generally useful for considering the growth of effective use of any mathematically-able software. The two elements of the personal aspect have generally applicability, as positive attitude and judicious use apply for learning to use any software tool. Many other software tools share with CAS the potential for both doing mathematics and helping students learn mathematics, so the common instances must reflect these dual functional and pedagogical purposes. The elements of the technical aspect are more specific to the application being targeted, but there is a useful structure here that can be generalised as: giving attention to fluent program syntax within modules, moving between modules of the software, and interpreting output. In this way, the structure dividing the technical and personal aspects into five elements should be useful to develop frameworks for monitoring progress and planning teaching with other mathematical software tools. 


\section{ACKNOWLEDGMENT}

The authors wish to thank the reviewers for their interest in this paper. Their detailed questions and comments gave us direction for revisions that have led to the paper's improvement.

\section{REFERENCES}

Arcavi, A. (1994). Symbol sense: informal sense-making in formal mathematics. For the Learning of Mathematics, 14(3), 24-35.

Arnold, S. (1995). Learning use new tools: A study of mathematical software use for the learning of algebra. Unpublished Ph.D. thesis, University of New South Wales.

Atkins, N., Creegan, A., \& Soan, P. (1995). You can lead students to DERIVE, but can you make them think? International DERIVE Journal, 2(1), 63-82.

Drijvers, P. (2000). Students encountering obstacles using CAS. International Journal of Computers for Mathematical Learning, 5, 189-209

Guin D. \& Trouche, L. (1999). The complex process of converting tools into mathematical instruments: the case of calculators. International Journal of Computers for Mathematical Learning, 3, 195-227.

Heid, M. K. (1988). Resequencing skills and concepts in applied calculus using the computer as a tool. Journal for Research in Mathematics Education, 19(1), 3-25.

Hillel, J. (1993). Computer algebra systems as cognitive technologies: implications for the practice of mathematics education. In C.Keitel and K.Ruthven ( Eds.) Learning from computer mathematics education and technology (pp. 18-47). Berlin: Springer-Verlag.

Kendal, M., \& Stacey, K. (2001). The impact of teacher privileging on learning differentiation with technology. International Journal of Computers for Mathematical Learning. 6(2), 143-165.

Kutzler, B. (1994). DERIVE - the future of teaching mathematics. International DERIVE Journal, 1(1), $37-48$.

Kutzler, B. (1999). The Algebraic Calculator as a Pedagogical Tool for Teaching Mathematics http://b.kutzler.com/article/art_paed/ped-tool.html 
Lagrange, J-B. (1996). Analysing actual use of a computer algebra system in the teaching and learning of mathematics. International DERIVE Journal, 3(3), 91-108.

Lagrange, J-B. (1999a). Techniques and concepts in pre-calculus using CAS: A Two year classroom experiment with the TI-92. The International Journal of Computer Algebra in Mathematics Education, 6(2), 43-65.

Lagrange, J.-B. (1999b). Complex calculators in the classroom: theoretical and practical reflections on teaching pre-calculus. International Journal of Computers for Mathematical Learning, 4, 51-81.

McIntosh, A., Reys, B. J., \& Reys, R. E. (1992). A proposed framework for examining basic number sense. For the Learning of Mathematics, 12(3), 2-8.

Pierce, R. (1999a). Using CAS as scaffolding for calculus: some observations. In W. Spunde, P. Cretchley, and R. Hubbard.(Eds.), The Challenge of Diversity: (Proceedings of the Delta- 99 Symposium on Undergraduate Mathematics, pp. 172-176). Brisbane: Delta 99 committee.

Pierce, R. (1999b). Computer algebra systems facilitate positive learning strategies. In J. Truran and K. Truran (Eds.), Making the difference. (Proceedings of the $22^{\text {nd }}$ annual conference of the Mathematics Education Research Group of Australasia, Adelaide, pp.431-438). Sydney: MERGA.

Pierce, R. (2002). An exploration of algebraic insight and effective use of computer algebra systems.

Ph.D. thesis. University of Melbourne. http://adt1.lib.unimelb.edu.au/adt-root/public/adtVU2003.0004/index.html

Pierce, R., \& Stacey, K. (2001a). Reflections on the changing pedagogical use of computer algebra systems: assistance for doing or learning mathematics. Journal of Computers in Mathematics and Science Teaching, 20(1), 141-163.

Pierce, R. \& Stacey, K. (2001b). Observations on students' responses to learning in a CAS environment. Mathematical Education Research Journal, 3(1), 28-46.

Pierce, R. \& Stacey, K. (2002). Algebraic insight: the algebra needed to use computer algebra systems. The Mathematics Teacher, 95(8), 622-627.

Verillon, P. \& Rabardel, P. (1995). Cognition and artifacts: A contribution to the study of thought in relation to instrumented activity. European Journal of Psychology in Education. 9(3), 77-101.

Weimer, R., (1998). Applied Calculus with Technology. Pacific Grove, U.S.A.: Brookes/Cole Publishing Co. 